\title{
A New Policy Based Management of Mobile IP Users
}

\author{
Hakima Chaouchi and Guy Pujolle \\ University of Paris VI, LIP6 networks Lab. \\ 8 rue du capitaine Scott, 75015 Paris \\ \{Hakima.CHAOUCHI ; Guy.pujolle\}@lip6.fr
}

\begin{abstract}
A policy based management networking is a new paradigm used to achieve the network management. This paper presents a new policy based Mobile IP users management architecture based on a Common Open Policy Service (COPS) protocol which is currently deployed for QoS management. This paper introduces a new concept of terminal policy enforcement point (TPEP) which allows the terminal to interact with the network enforcing network policies defined by the network manager; it is a key feature of our architecture. The paper presents also the global architecture to support the mobile IP users requirements based mainly on two extensions of COPS protocol; COPS-SLS [1] for QoS negotiation and COPS-MU/MT [2] for policy based user and terminal mobility management.
\end{abstract}

\section{Introduction}

Due to the tremendous success of IP technology in the fixed network area, it is commonly accepted today that IP will provide the unifying glue for the increasingly heterogeneous, ubiquitous, and mobile environment [3, 4]. This paper presents new policy based architecture for user mobility management which supports nomadic users in the Internet by allowing them to access their personalized computing resources and services from anywhere on the Internet [5].

The IETF has proposed a policy based model for network management $[6,7,8]$ and a TCP based policy transport protocol, called COPS (Common Open Policy Service) [9]. Policy based network management currently concerns QoS and security management. Many extensions have been introduced for COPS usage such as COPSPR [9] for Diffserv, COPS-RSVP [10] for Inserv, and COPS-MIP [11] for Mobile IP terminal mobility management.

The next section presents an overview of a user mobility aspect and an overview of a policy based management networking. Then a new architecture to support Mobile IP users' management is presented followed by a conclusion.

\subsection{User Mobility Overview}

User mobility concerns terminal mobility and personal mobility. The Terminal mobility allows a terminal to change its network point of attachment without being disconnected from the network [12]. IP networks support terminal mobility using 
Mobile IP protocol. Personal mobility allows a user to use any available mobile or fixed terminal, and use his personal subscribed home network services from any terminal and any network access [13]. Thus, personal mobility is related to user location and service portability management [14]. A universal personal identifier is necessary to achieve personal mobility.

\subsection{Policy Based Architecture Overview}

Policy based management networking (PBMN) framework is proposed by the IETF $[6,7]$. It is based on two important elements: policy server PDP (Policy Decision Point) and PEP (Policy Enforcement Point) as illustrated on Fig. 1. (a). PBMN intends to manage the network based on the business policies, these policies are translated to network policies and stored in the network. They are used to automatically configure the network elements to offer services based on the business level policies. The protocol used to exchange policy objects is COPS [9]. PDP and PEP exchange COPS messages which are detailed in [15] to achieve policy based network management. Fig. 1 (b) illustrates an example of PDP/PEP messages exchange process which are briefly explained below:

OPN: Client OPeN, PEP opens a TCP connection with the PDP; CAT: Client AccepT, the PDP accepts a connection; REQ: REQuest. PEP sends a request for a PDP. The request contains an identifier and policy objects necessary for a PDP policy decisions; DEC: DECision. The PDP sends a policy decision in a DEC message; RPT: RePorT. The PEP sends a report to the PDP after enforcing a policy contained in previous DEC message; CC: Client Close. The PEP closes a connexion.
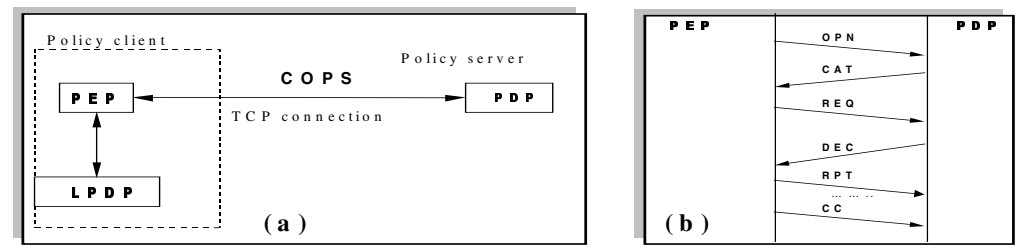

Fig. 1. (a) Policy based architecture. (b) PDP/PEP COPS messages exchange.

\section{A New Policy Based Mobile IP Users' Management Architecture}

We identify four issues related to user mobility management which are user registration, terminal registration, service portability and QoS negotiation.

To achieve these challenging issues, we introduce new components in the IETF policy based architecture illustrated on Fig. 2 and we introduce COPS extension called COPS-MU/MT [2] (COPS-Mobile User/Mobile Terminal) which defines new policy objects to support user and terminal registration respectively, user service portability, and QoS negotiation. 


\subsection{Architecture Components}

Some Mobile IP terms [16] are necessary to understand the next sections, they are explained bellow:

HA. Home Agent, maintains a mobility binding of a MT in his home network.

FA. Foreign Agent maintains a list of terminal visitors in the foreign network.

Mobility binding. It's an association between the Home address and the CoA of a mobile terminal;

Home address. It's a routable and a permanent address used to locate a mobile terminal even when it changes its point of attachment. It is a HA adress.

CoA. Care of Address, is the address obtained in a foreign network. CoA may be a FA address (IPv4) or a co-located CoA (IPv6) [16]. If a mobile terminal has a colocated CoA, it interacts directly with the HA else, it interacts with the FA which forwards its messages to the HA.

Fig. 2 illustrates new components introduced in COPS-MU/MT architecture.
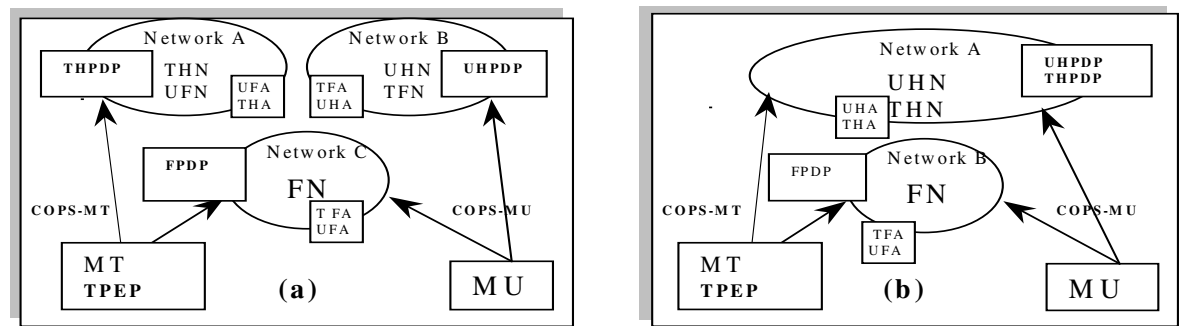

Fig. 2. Terminal's and user's home and foreign networks. (a) MU and MT are subscribed in different networks. (b) MU and MT are subscribed in the same network.

Some components defined for a Mobile User (MU) and a Mobile Terminal (MT) have similar functions such as a:

A User Home Policy Decision Point (UHPDP) and a Terminal Home Decision Point (THPDP) which are policy servers in a User Home Network (UHN) and a Terminal Home Network (THN) respectively.

A User Foreign Policy Decision Point (UFPDP) and a Terminal Foreign Policy Decision Point which are policy servers of a User Foreign Network (UFN) and a Terminal Foreign Network (TFN) respectively.

A Foreign policy Decision Point (FPDP) is a policy server of a Foreign Network (FN) of a mobile user and a mobile terminal.

A key feature of our architecture is a Terminal Policy Enforcement Point (TPEP). It is introduced to allow the terminal to interact directly with the network for user and terminal registration, QoS negotiation and user service portability.

User Home Agent (UHA) and Terminal Home Agent (THA) maintains the user and the terminal mobility binding respectively.

User Foreign Agent (UFA) and Terminal Foreign Agent (TFA) are the FA of the mobile user and the mobile terminal respectively.

Policy servers of different network providers have to maintain policy information related to home and foreign mobile users such as user profile, services profile, and 
terminal profile in order to allow a user universal access to network services and resources from anywhere. The different profiles may be stored in the home agents or in the policy servers.

The goal of the policy based Mobile IP user management is to allow the user to access his home services with the parameters negotiated with his home network from anywhere. Thus, the policy based Mobile IP users management achieves the user and terminal registration to support user and terminal location management and the personal service portability and the QoS negotiation to support user services anywhere.

\subsection{Policy Based User and Terminal Registration}

Terminal registration. Terminal registration must be achieved only if a terminal is located in a foreign network, if it is a fixed terminal or a mobile terminal located in its home network then a terminal registration is unnecessary. COPS-MT is used to achieve the terminal registration, it supports IPv4 and IPv6 registration by allowing the TPEP directly interact with the FPDP so that the mobile terminal can achieve its registration directly with the HA. Fig. 5 illustrates COPS-MT terminal registration related to FA CoA (IPv4) and co-located CoA (IPv6).

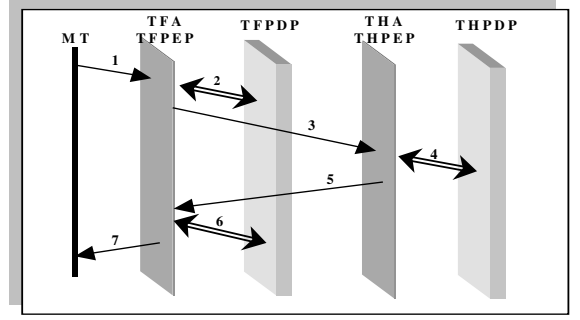

(a)

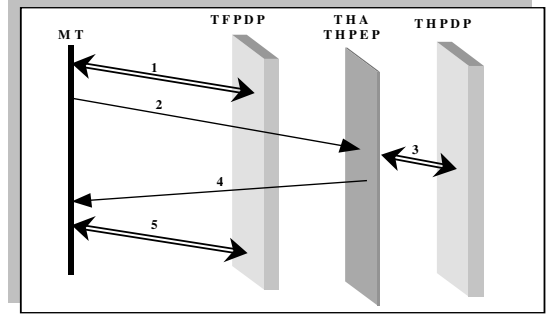

(b)

Fig. 3. COPS-MT terminal registration. (a) FA CoA. (b) Co-located CoA.

Numbered steps illustrated on Fig. 3 (a) correspond to the terminal registration in Mobile IPv4 with FA CoA whereas Fig. 3 (b) corresponds to the case when a mobile terminal has a co-located CoA such as in IPv6. Fig. 3 (b) steps are explained bellow:

1. TPEP interacts directly with TFPDP for terminal registration request policy decisions;

2. MT sends registration request to the THA;

3. THPEP interacts with THPDP for terminal registration request;

4. THA sends registration reply message to the MT;

5. TPEP interacts with FPDP for terminal registration reply policy decisions.

The steps described on Fig.3 (a) are related to the case where a MT has a FA CoA, they are different from steps in Fig.3 (b) in that a FA intercepts messages sent by a MT and forwards them. 
User registration. A user registration must be achieved every time a user logs in a terminal even if a user is in his home network. In COPS-MU, the user mobility registration is similar to COPS-MT terminal mobility registration. COPS-MU user registration consists of maintaining an association between the terminal home adress and a user identifier. The necessary elements for achieving user registration are UHPDP, UFPDP, UHA and UFA. The registered user would be reachable on the terminal he is using and may use his home services from anywhere.

\subsection{Policy Based Mobile IP User Service Portability and QoS Negotiation}

In this work we assume that the network is a policy based network QoS management such as Diffserv COPS-PR policy provisioning based network and we propose to support a QoS negotiation for a Mobile IP user which moves from the home network to a foreign network. In this architecture COPS-MU/MT is deployed in a wireless access network to achieve a user and terminal registration and QoS negotiation, and COPS-SLS [1] is deployed between the home PDP and a FPDP for inter-domain negotiation of a user home subscribed QoS and COPS-MU for inter-domain mobile user and mobile terminal registration and mobile user service portability negotiation.

When a mobile user moves to a foreign network, a FPDP interacts with the UHPDP to determine the user's QoS negotiated with the home network so that the mobile user has not to re-negotiate a QoS with the foreign network. This architecture is illustrated in Fig. 4.

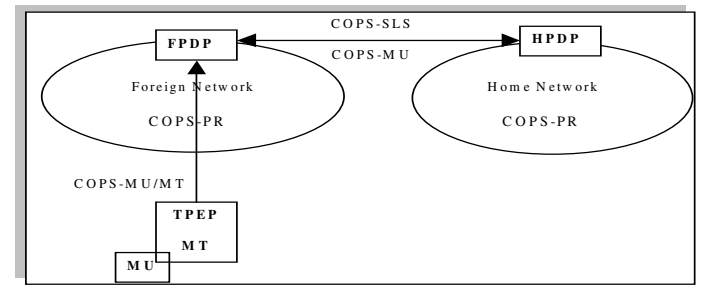

Fig. 4. Policy based Mobile IP users QoS negotiation environment.

This architecture is also used to negotiate user service portability. The FPDP negotiate with the UHPDP where to run the user personal services. The UHPDP decides based on the user profile, the personal service profile and the terminal profile where to run the user personal service. This part will not be detailed in this paper.

\section{Conclusion}

In this paper, we have described new policy based architecture to support user mobility management in IP networks. The approach taken assumes that mobile users are in IP networks based on the PDP/PEP architecture and using COPS protocol. We have proposed to use a terminal Policy Enforcement Point (TPEP) which allows the terminal to interact directly with the appropriate PDP and we proposed also the COPS 
extension named COPS-MU/MT (COPS-Mobile User/Mobile Terminal) to support a policy based user mobility management issues related to user and terminal registration, user services portability and QoS negotiation.

We believe that the use of COPS and PDP/ PEP model offers a good way to achieve a unified IP network policy management of QoS, security, mobility, etc. However, we need to implement this architecture for performance evaluation.

Future work intends to define all necessary policy objects related to user mobility registration, terminal mobility registration, service portability, and QoS negotiation.

\section{References}

[1] M. Nguyen, "COPS-SLS", IETF-draft, november 2001, draft-nguyen-rap-cops-sls-01.txt

[2] H.CHAOUCHI, G. Pujolle, «COPS-MU : a new policy based user mobility management », proceeding MS3G 2001, Lyon, France.

[3] A. Fasbender,F. Reichert, E. Gueulen, J. Hjelm, T. Wierelemann, “Any Network, Any Terminal, Anywhere", IEEE Personal Communications 1999.

[4] L. Bos, S. Leroy, "Toward an ALL-IP-Based UMTS System Architecture", IEEE Network, January/February 2001.

[5] Yalun Li, V. Leung, "Protocol Archirtecture for universal personal computing", IEEE Journal on selected areas in communications, vol 15, $\mathrm{N}^{\circ}$ 8, October 1997.

[6] A. Wtersinen, J. Schnizelein, J;Strassner, M. Scherling, B. Quinn, J. Perry, S. Herzog, A. Huynh, M. Carlson, S. Waldbusser, "Policy Terminology", Internet Draft, March 2001, draft-ietf-policy-terminology-02.txt

[7] B. MOORE, E. Ellesson, J. Strassner, A. Westerinen, "Policy Core Information Model", RFC 3060, February 2001.

[8] M. Fine, K. McCloghrie, J. Seligson, K. Chan, S; Hahn, R. Sahita, A. Smith, F. Reichmeyer, "Framework Policy Information Base", Internet draft, March 2001, draftietf-rap-frameworkpib-04.txt.

[9] K. Chan, J. Seligson, D. Durhan, K. Mcloghrie, S. Herzog, F. Reichmeyer, R. Yavatkar, A. Smith "COPS usage for Policy provisioning (COPS-PR)", RFC 3084, March 2001.

[10] S. Herzog, J Boyle, R.Cohen, D.Durham, R.Rajan, A.Sastry, "COPS usage for RSVP", RFC 2749, January 2000.

[11] M. Jaseemuddin, A. Lakas, 'COPS usage for Mobile IP ', Internet draft, October 2000, draft-ietf-jaseem-rap-cops-mip-00.txt.

[12] G. Forman, J. Zahorjan, " the challenge of mobile computing", IEEE Computer, March 1994.

[13] E. Koukoutsis, C. Kossidas, N. Polydorou, “ User Aspects for Mobility”, Acts Guideline SII-G8/0798.

[14] M. Cristina Ciancetta, G. Colombo, R. Lavagnolo, D. Grillo, F. Bordoni, "Convergence Trends for fixed and mobile services", IEEE Personnal Communications, April 1999.

[15] D. Durham, J. Boyle, R. Cohen, S. Herzog, R. Rajan, A. Sastry, "The COPS (Common Open Policy Service) Protocol", RFC 2748, January 2000.

[16] C. Perkins, "IP mobility support", RFC 2002, October 1996. 